\title{
WAVELET DECOMPOSITION AND ALPHA STABLE FUSION
}

\author{
Rachid Sabre ${ }^{1}$ and Ias Wahyuni ${ }^{2}$ \\ ${ }^{1}$ Laboratory Biogéosciences CNRS, University of Burgundy/Agrosup Dijon, France \\ ${ }^{2}$ Universitas Gunadarma, J1. Margonda Raya No 100 Depok 16424, Indonesia
}

\begin{abstract}
This article gives a new method of fusing multifocal images combining the Laplacian pyramid and the wavelet decomposition using the stable distance alpha as a selection rule. We start by decomposing multifocal images into several pyramid levels, then applying the wavelet decomposition to each level. the originality of this work is to use the stable distance alpha to fuse the wavelet images at each level of the Pyramid. To obtain the final fused image, we reconstructed the combined image at each level of the pyramid. We compare our method to other existing methods in the literature and we deduce that it is almost better.
\end{abstract}

\section{KEYWORDS}

Image fusion, Laplacian pyramid, Wavelet decomposition

\section{INTRODUCTION}

The principle of image fusion is the combination of relevant information from two or more images into a single image giving all the information carried by the input images. The purpose of image fusing is to reduce the blurred area and maximize the bright area in the output image so it increases the information relevant to an application or task. New technology uses images taken by several tools to be sure of interpretation. So it is possible to obtain information from multiple images hence the need to fuse them to obtain high quality information. The images discussed in this article are multifocal. Due to the limited depth of field of optical lenses in cameras, it is often not possible to get an image containing all the relevant objects " in focus " so that a scene can be broken down into a whole images with different focus for each image. in this case we use the image fusion method to get all the focused objects.

In the literature, many methods for performing image fusion are studied [1] - [3]. This work uses the Laplacian pyramid (LP) and transforms it into discrete wavelets (DWT). These two methods consist in fusing the transformation images at several levels.

The fusion of LP images between multi-source information at the basic level to give precise and reliable information. The choice of selection rule to determine the value of each pixel in the resulting fused pyramid is very important in fusion LP images. Among the selection rules used in LP image fusion are the average method, the maximum method, the salience and the correspondence measure [4], and the combination of the average and maximum energy method [4]. Recently, [6] used PCA as a selection rule in LP image fusion and wavelet decomposition with the maximum absolute selection rule [7].

On the other hand, the wavelet fusion method decomposes the image into different types of subbands of coefficients. The fusion of images using the wavelet method can be seen in [8] - [11]. 
Signal \& Image Processing: An International Journal (SIPIJ) Vol.11, No.1, February 2020

The edge characteristics are well preserved with the maximum wavelet transformation module. Thus, the information on the components of the object in a new fused image preserving the detailed information on the image [7].

The new method of fusing multifocal images that we propose in this work uses the Laplacian pyramid (LP) and the wavelet transform with a fusion by alpha stable distance as a selection rule. It begins with the decomposition into discrete wavelets at each level of LP. Then it fuses the wavelet coefficients by a model based on the alpha stable distance. This method of fusion contributed significantly to the improvement of the resulting fused image. This paper is organized as follows: Section 2 briefly gives the construction of the stable alpha distance of the neighbouring pixels and the model of the fusion. Section 3 develops the proposed method. Section 4 is an application of the proposed method on real images and comparison with other methods. Section 5 presents our conclusions and identifies potential perspectives.

\section{Neighbouralpha Stable Distance And Fusion}

Stable alpha laws are widely used in the processing of impulsive or spiky signals. It has been used for example in [12] and [13] to give the model of sea congestion in SAR images using a stable alpha distribution for the detection of ships, in [14] the noise of speckle a been removed using the Bayesian algorithm based on stable alpha in the wavelet domain. The stable alpha distribution is also used in image segmentation [15] as well as in compression image fusion [12]. [14], [15], [13]. We then give a brief overview of the alpha-stable distribution.

\section{A. Neighbor alpha stable distance}

This work propose a novel fusion method using a new model as a selection rule in Laplacian pyramid method. This consisted of weighting each pixel of each image by exponential of Neighbor Alpha Stable Distance (NASD). This neighbor alpha stable distance generalize the quadratic difference between the value of the pixel $(\mathrm{x}, \mathrm{y})$ and the all pixel values of its neighbors when . The neighbor, with the size "a", of a pixel (x,y) is defined as follows:

$(x+i, y+j)$ where $i=-a,-a+1, \ldots, a-1, a$ and $j=-a,-a+1, \ldots, a-1, a$.

For example the neighbour with the small size ("a" $=1)$ contains: $(x-1, y-1),(x-1, y)$, $(x-1, y+1),(x, y-1),(x, y+1),(x+1, y-1),(x+1, y),(x+1, y+1)$.

\begin{tabular}{|l|l|l|l|}
\hline & & \\
\hline & $(\mathrm{x}-1, \mathrm{y}-1)$ & $(\mathrm{x}-1, \mathrm{y})$ & $(\mathrm{x}-1, \mathrm{y}+1)$ \\
\hline & $(\mathrm{x}, \mathrm{y}-1)$ & $(x, y)$ & $(\mathrm{x}, \mathrm{y}+1)$ \\
\hline & $(\mathrm{x}+1, \mathrm{y}-1)$ & $(\mathrm{x}+1, \mathrm{y})$ & $(\mathrm{x}+1, \mathrm{y}+1)$ \\
\hline & & & \\
\hline & & & \\
\hline
\end{tabular}

Figure 1. Pixel at $(x, y)$ within its neighborhood, $a=1$.

Then, the steps of image fusion with size " $a$ "wereas follows: 
Signal \& Image Processing: An International Journal (SIPIJ) Vol.11, No.1, February 2020

Let $I_{1}, \ldots, I_{M}$ be $M$ original source images with different focus to fuse.The images here had the same size $\left(N_{1} \mathrm{x} N_{2}\right)$. The general principle of making fusion rules were:

Step 1: For each pixel of each image, we calculated the neighbour alpha stable distance (NASD) of every source image, $d_{a, k}(x, y)$ :

$$
d_{a, k}(x, y)=\left(\frac{1}{R} \sum_{m=-a}^{a} \sum_{n=-a}^{a}\left|I_{k}(x, y)-I_{k}^{\prime}(x+m, y+n)\right|^{\alpha}\right)^{\frac{1}{\alpha}}
$$

Where

$$
\begin{aligned}
& I_{k}^{\prime}(x+m, y+n)=\left\{\begin{array}{l}
I_{k}(x+m, y+n), \text { if } 1 \leq x+m \leq N_{1} \text { and } 1 \leq y+n \leq N_{2}, A=(2 a+1)^{2}-\operatorname{card}(S), \\
I_{k}(x, y), \text { otherwise }
\end{array}\right. \\
& S=\left\{(m, n) \in\left([-a, a]^{2}-\{(0,0)\}\right) \mid I_{k}^{\prime}(x+m, y+n)=I_{k}(x, y)\right\} . k=1, \ldots, M .
\end{aligned}
$$

\section{B. Neighbor Alpha Stable Fusion}

The Model of the fusion based on Neighbour Alpha Stable Fusion (NASF) is:

$$
\operatorname{NASF}(x, y)=\frac{\sum_{i=1}^{M} \exp \left(d_{a, k}(x, y)\right) I_{i}(x, y)}{\sum_{i=1}^{M} \exp \left(d_{a, k}(x, y)\right)}
$$

Obviously, this method depended on the size "a". We have noticed that,for almost all images, $a=4$ gave the best result. In the following we took $a=4$.

\section{The Proposed Method}

First, we decomposed each source image by Laplacian pyramid (LP). The paper [16] introduced (LP) as a binocular fusion model in human stereoscopic vision. The selection rule used was the maximum at each level of the pyramidal transformation [17]. Each level of the Laplacian pyramid has been built recursively from its lower level by applying the following four basic steps: blurring (low-pass filtering), subsampling (size reduction), interpolation (expansion) and differentiation (to subtract two image pixels per pixel). The lowest level of the pyramid corresponds to the original image.

At each level of Laplacian pyramid, we applied Decomposition Wavelet Transform (DWT).Using Haar wavelet transform. DWT separately filtered and lowered samples images in the horizontal direction and vertical directions. This gave four coefficient subbands at each scale. As presented in [7], we supposed an image $I(x, y)$ and denoted the horizontal frequency first by using 1-D lowpass filter $\mathrm{L}$ and highpass filter $\mathrm{H}$ produces the coefficient matrices $I_{L}(x, y)$ and $I_{H}(x, y)$ and then followed the vertical frequency second by using lowpass filter $\mathrm{L}$ and highpass filter $\mathrm{H}$ to each column in $I_{L}(x, y)$ and $I_{H}(x, y)$. Thisgave four sub-images $I_{L L}(x, y), I_{L H}(x, y)$, $I_{H L}(x, y)$, and $I_{H H}(x, y)$ for one level of decomposition. By recursively applying the same scheme to the low-low subband amultiresolution decomposition could be achieved. 
Signal \& Image Processing: An International Journal (SIPIJ) Vol.11, No.1, February 2020

Thus, we used the NASF fusion defined in (6) to fuse images in wavelet decomposition by fusing the DWT coefficient of every corresponding frequency band.

The fusion follows 4 steps: we supposed there were two original source images, A and B, with different focus:

1) Performing Laplacian pyramid decomposition to create Laplacian pyramid for each source image,

2) Performing discrete wavelet decomposition to every level of Laplacian pyramid for each image in different kinds of coefficient,

3) Fusing an appropriate coefficient of the corresponding subband to obtain new coefficients by using Neighbor Alpha Stable Fusion (NASF). The fused wavelet image is achieved through the inverse discrete wavelet transform,

4) The final fused image wasobtained by performing pyramid inverse transform on the fused wavelet image by using Neighbor Alpha Stable Fusion (NASF).

The process of pyramid image fusion can be seen in Fig.2, in which the fusion LPi's (i=1, 2, 3) wasobtained by applying wavelet transform and (NASF) fusion. Actually, it couldbe extended to more than twosource images A and B.

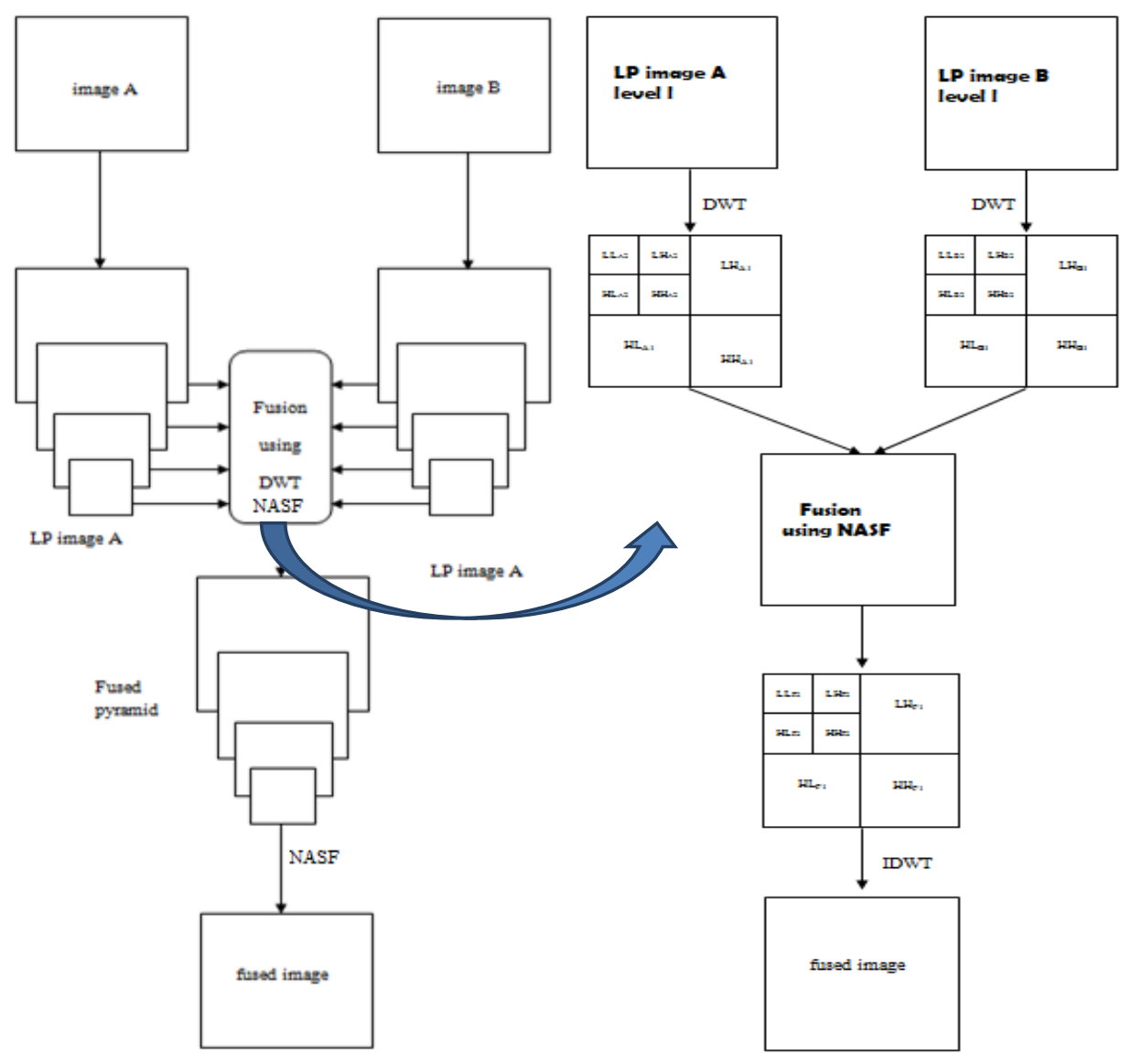

Figure 2. The proposed method Figure 3. DWT image fusion

The figure 3shows the process of decomposition wavelet transform of LP images at level $l$ and fusion image using NASF method. 


\section{EXPERIMENTAL RESULT}

As in the work [18], we have used in this section images from the database [12]. We have blurred an area of each image using the convolution of the Gaussian filter applied to the reference image gf: the choice of Gaussian is validated in the works [19] - [20]. The blurred zones are chosen to hide an object from the photographed scene when there are several objects. Thus, the size of the blurred areas varied according to the size of the objects hidden in the images.Let $g_{f}$ thereference image. The blurred image $g_{0}$ wasdefined as follows:

$g_{0}(i, j)=\left\{\begin{array}{l}\sum_{n=-2}^{2} \sum_{m=-2}^{2} h\left(m^{\prime}, n^{\prime}\right) g_{f}\left(i-m^{\prime}, j-n^{\prime}\right),(i, j) \in \text { blurred area } \\ g_{f}(i, j),(i, j) \in \text { object focus area }\end{array}\right.$

where $h\left(m^{\prime}, n^{\prime}\right)$ is Gaussian filter. We applied the method on 150 sets of multifocus images on a datasets of images [12]. This paper presented only five set of multi focus images. Figures4, 6, 8,10 and 12 show the multi focus images obtained by the convolution of Gaussian filter. Figures $5,7,9,11$ and 13 show the fused image by proposed method.Visually the image obtained by the proposed method gives a very satisfactory fusion.

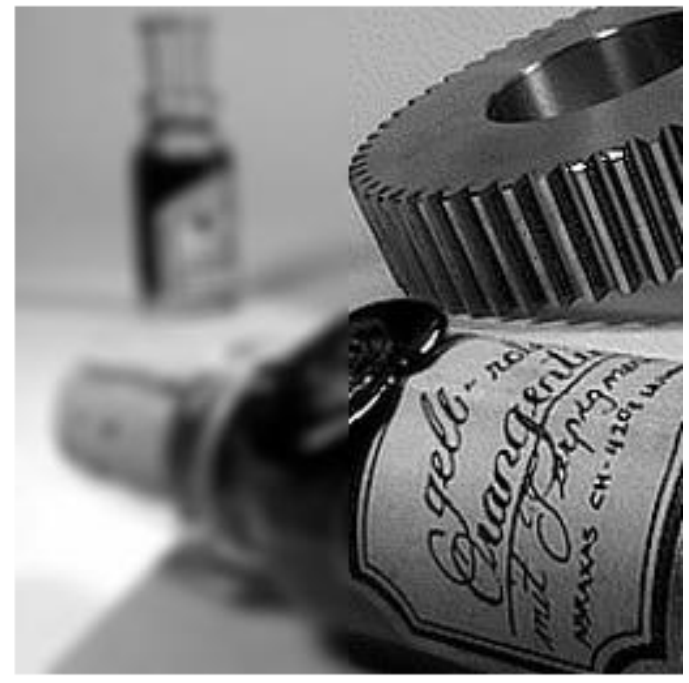

Blurred image1

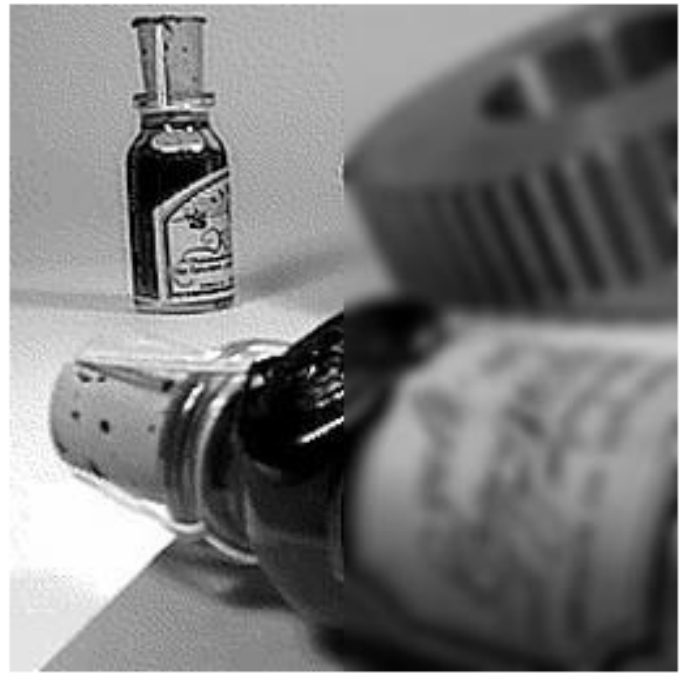

Blurred image2

Figure4. Multi focus images (bottles)

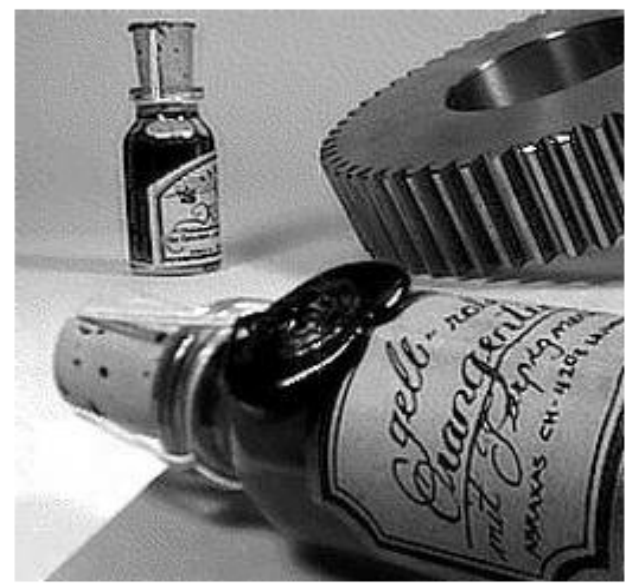

Figure5. Fused image by proposed method (bottles) 
Signal \& Image Processing: An International Journal (SIPIJ) Vol.11, No.1, February 2020

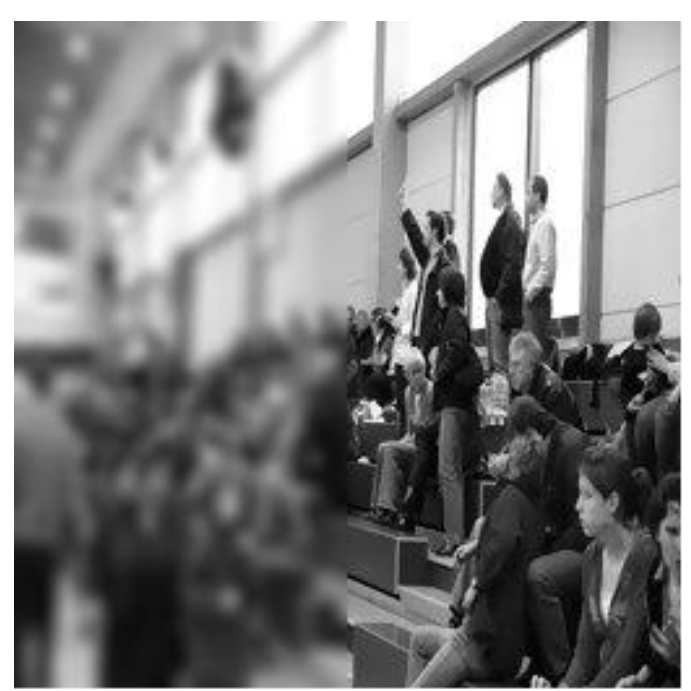

Blurred image 1

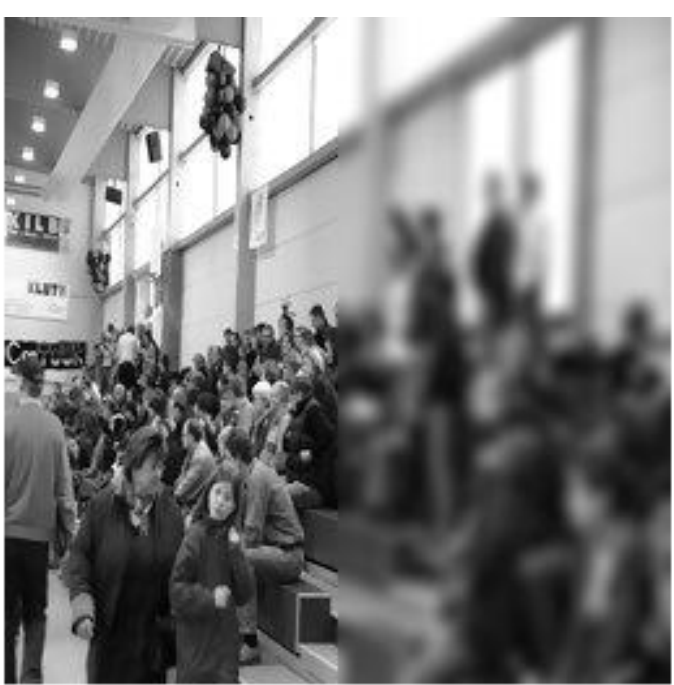

Blurred image2

Figure6. Multi focus images (people)

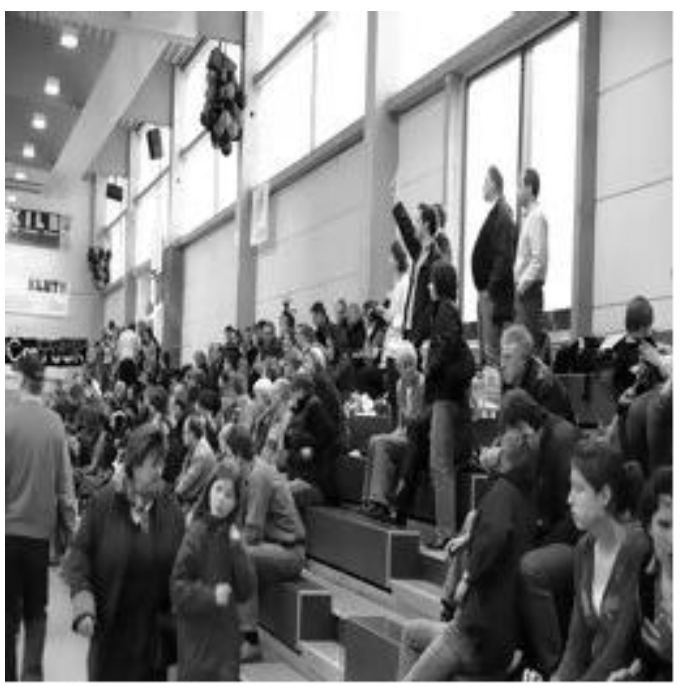

Fusion, proposed method

Figure7. Fused image by proposed method (people) 
Signal \& Image Processing: An International Journal (SIPIJ) Vol.11, No.1, February 2020

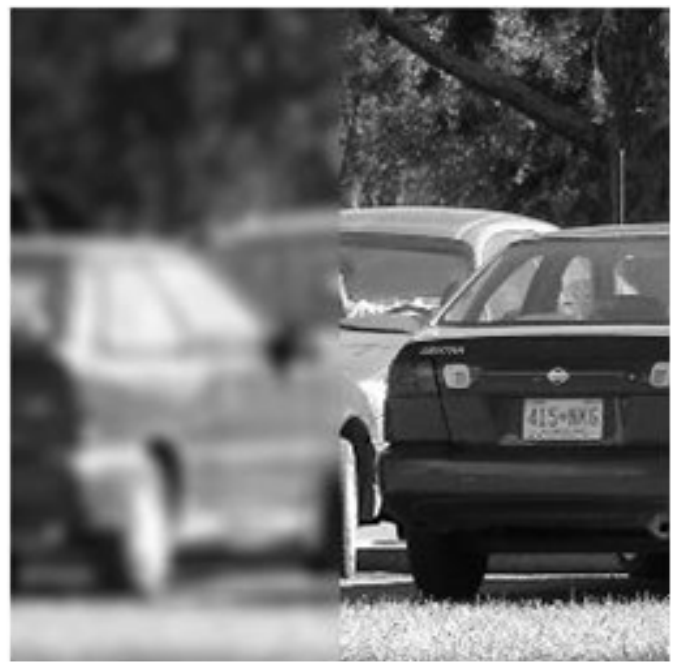

Blurred image1

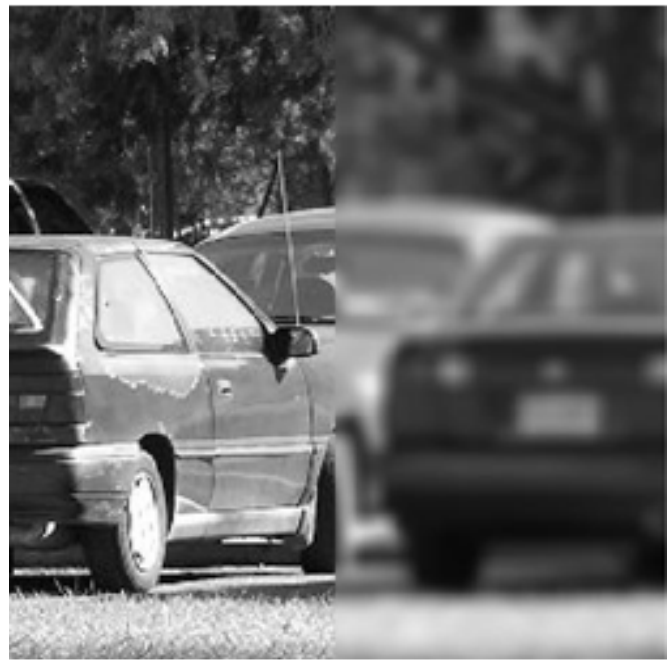

Blurred image2

Figure 8. Multi focus images (cars)

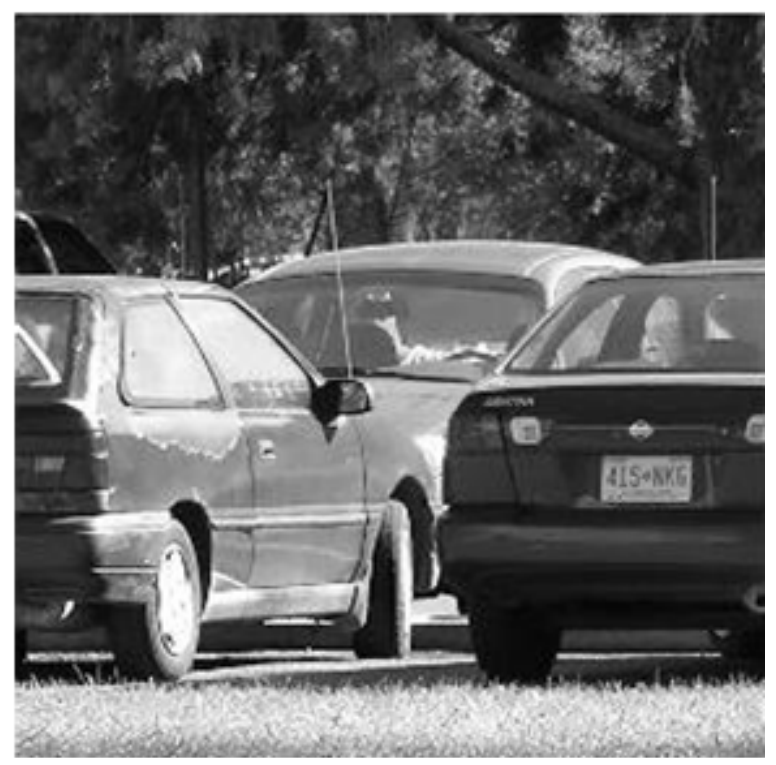

Image fused by proposed method

Figure 9. Fused image by proposed method (cars) 
Signal \& Image Processing: An International Journal (SIPIJ) Vol.11, No.1, February 2020

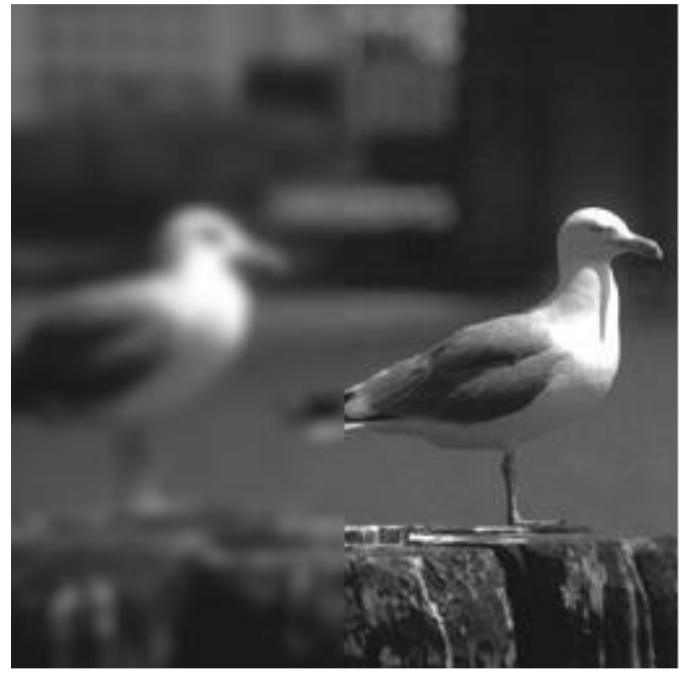

Blurred image1

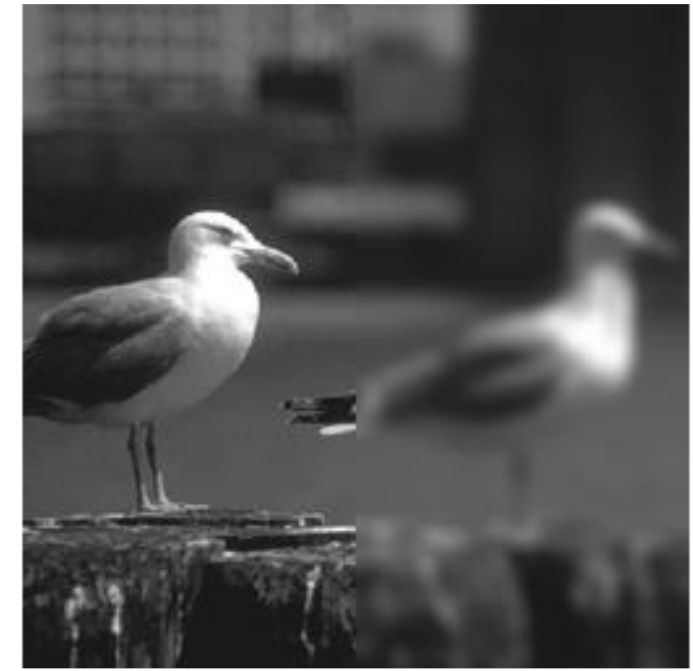

Blurred image2

Figure 10. Multi focus images (birds)

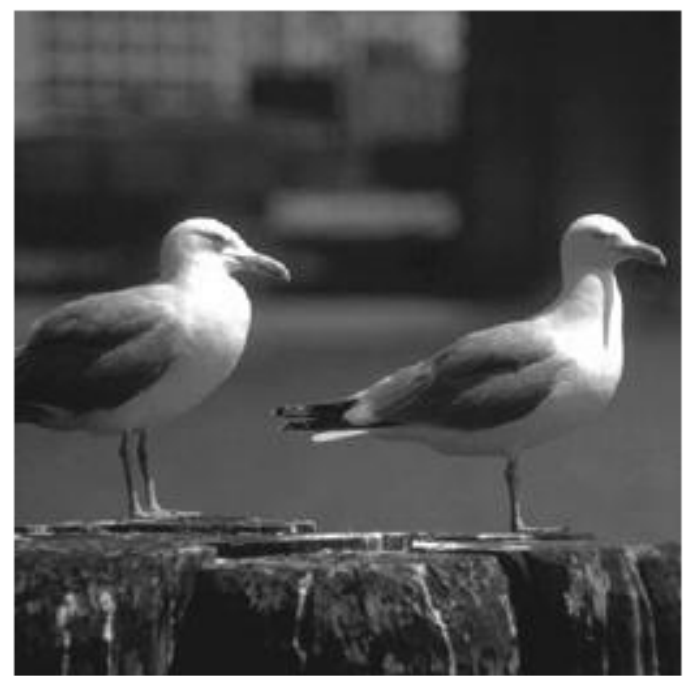

Image fused by proposed method (birds)

Figure 11. Fused image by proposed method (birds) 
Signal \& Image Processing: An International Journal (SIPIJ) Vol.11, No.1, February 2020

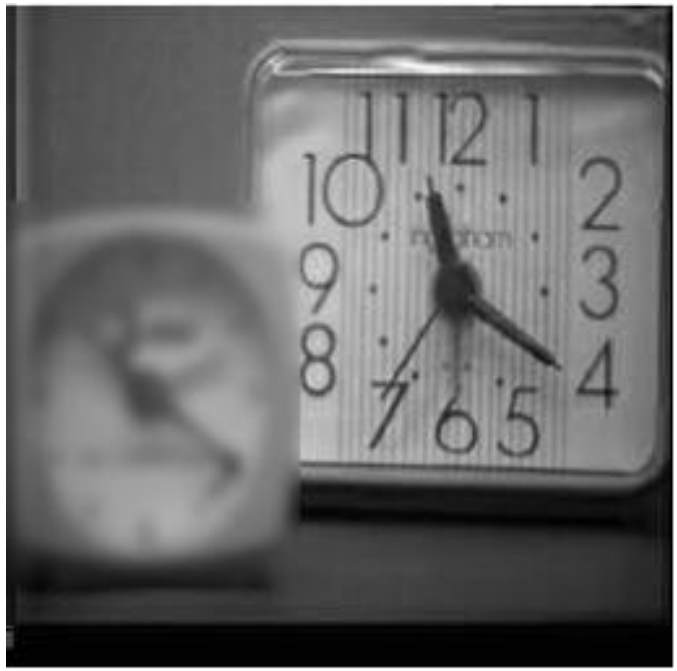

Blurred image1

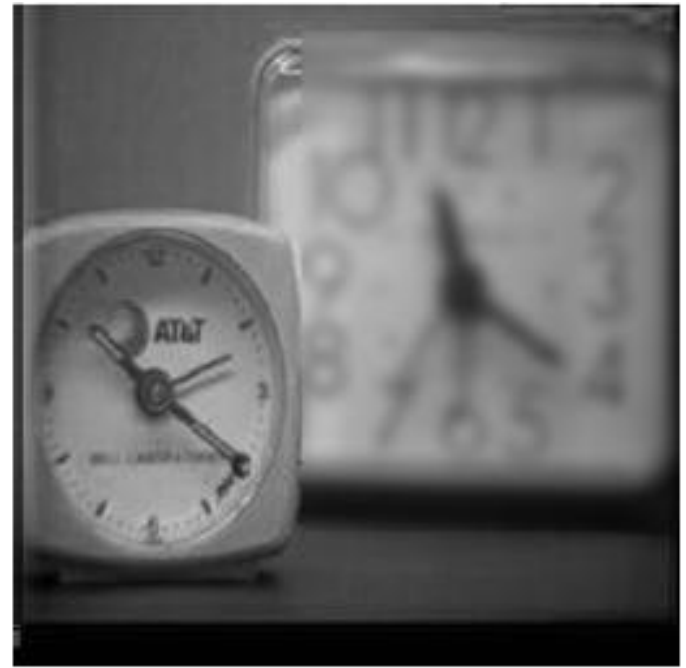

Blurred image2

Figure 12. Multi focus images (clocks)

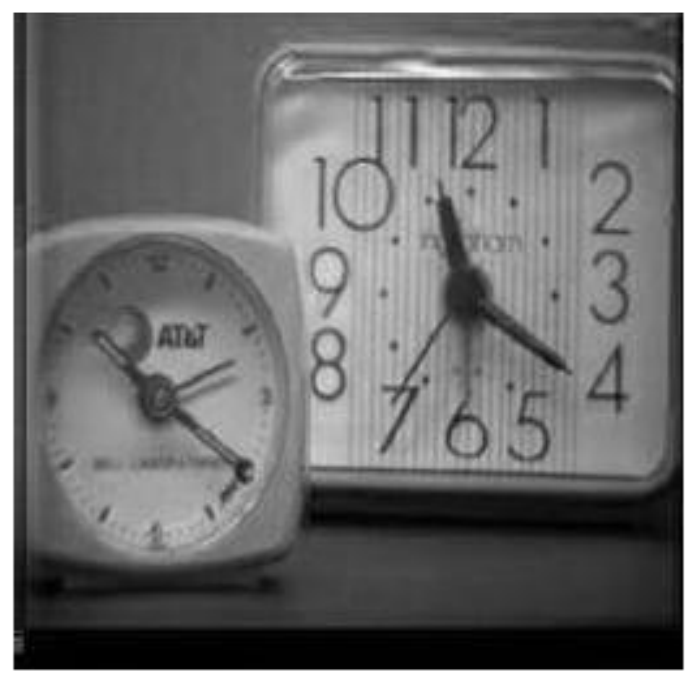

Image fused by proposed method (clocks)

Figure 13. Fused image by proposed method (clocks)

We compared the proposed method to other methods like: Principal Component Analysis (PCA) [5], Decomposition Wavelet Transform (DWT) [8]-[9] and [14]-[15], Bilateral Gradient-based (BG) [21], Laplacian Pyramid with Average as selection rule (LP_AV), Laplacian Pyramid with PCA as selection rule (LP_PCA) [22].

For that, we used the quantitative analysis root mean square error (RMSE) defined by:

$$
R M S E=\sqrt{\frac{1}{m n} \sum_{i=1}^{m} \sum_{j=1}^{n}[R(i, j)-F(i, j)]^{2}}
$$


Signal \& Image Processing: An International Journal (SIPIJ) Vol.11, No.1, February 2020 where $F(i, j)$ is the gray level intensity of pixel $(i, j)$ of the fused image and $R(i, j)$ is the gray level intensity of pixel $(i, j)$ of the reference image.

A smaller value of RMSE corresponds to a good fusion result. If the value of RMSE The mean and standard deviation of RMSE for the studded methods. Are given in the table 1

Table 1. Statistic parameters of the sample (150 images)

\begin{tabular}{|l|l|l|l|l|l|l|}
\hline Method & LP_AV & PCA & BG & LP_PCA & DWT & Proposed_method \\
\hline Mean & 8,7452 & 8,7143 & 11,0449 & 8,6043 & 4,1941 & 2,1589 \\
\hline $\begin{array}{l}\text { Standard } \\
\text { deviation }\end{array}$ & 3,8810 & 3,8672 & 4,8593 & 3,8549 & 1,3813 & 0,9437 \\
\hline
\end{tabular}

The proposed method has a smaller mean of the RMSE $(2,1589)$. The following histograms of RMSE for 150 imagescorrespondingdifferent methods (Figure 14, 15, 16,17,18 and 19) showed that for all methods the values of RMSE werealmost symmetrically centered around the mean value.

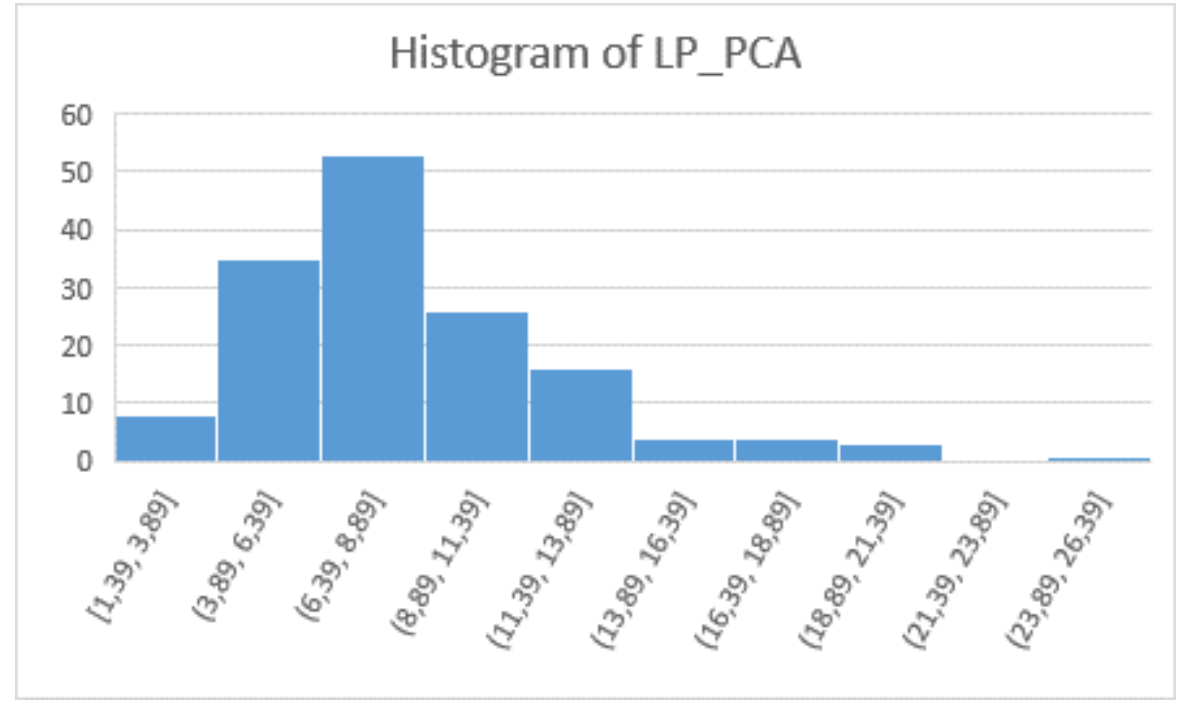

Figure 14. The histogram of LP_PCA method

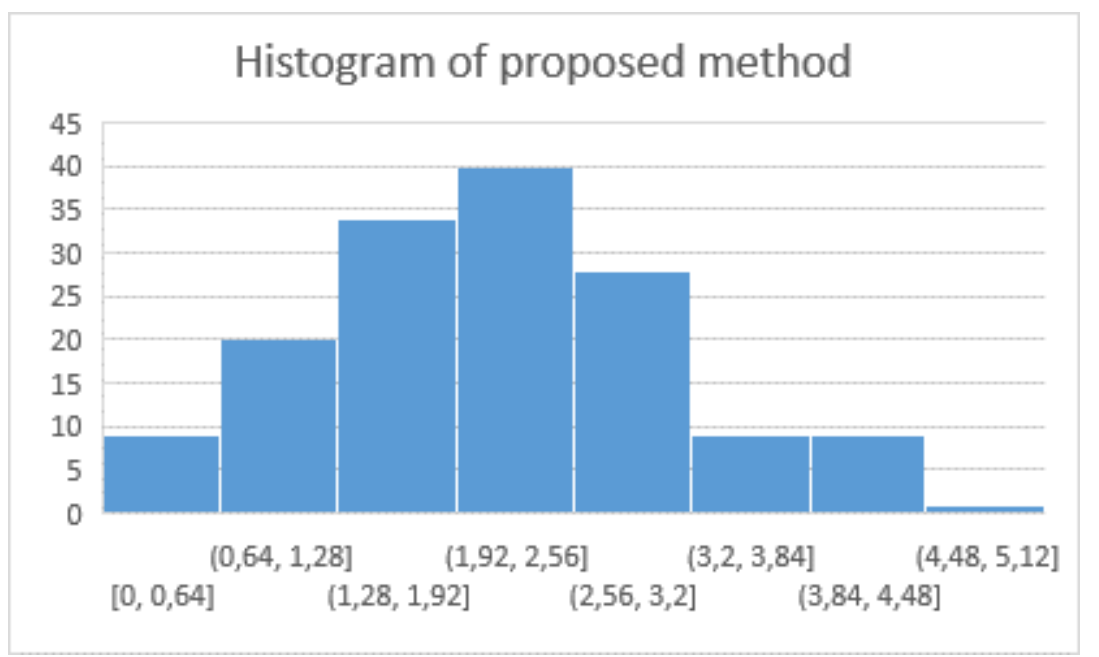

Figure 15. The histogram of proposed method 
Signal \& Image Processing: An International Journal (SIPIJ) Vol.11, No.1, February 2020

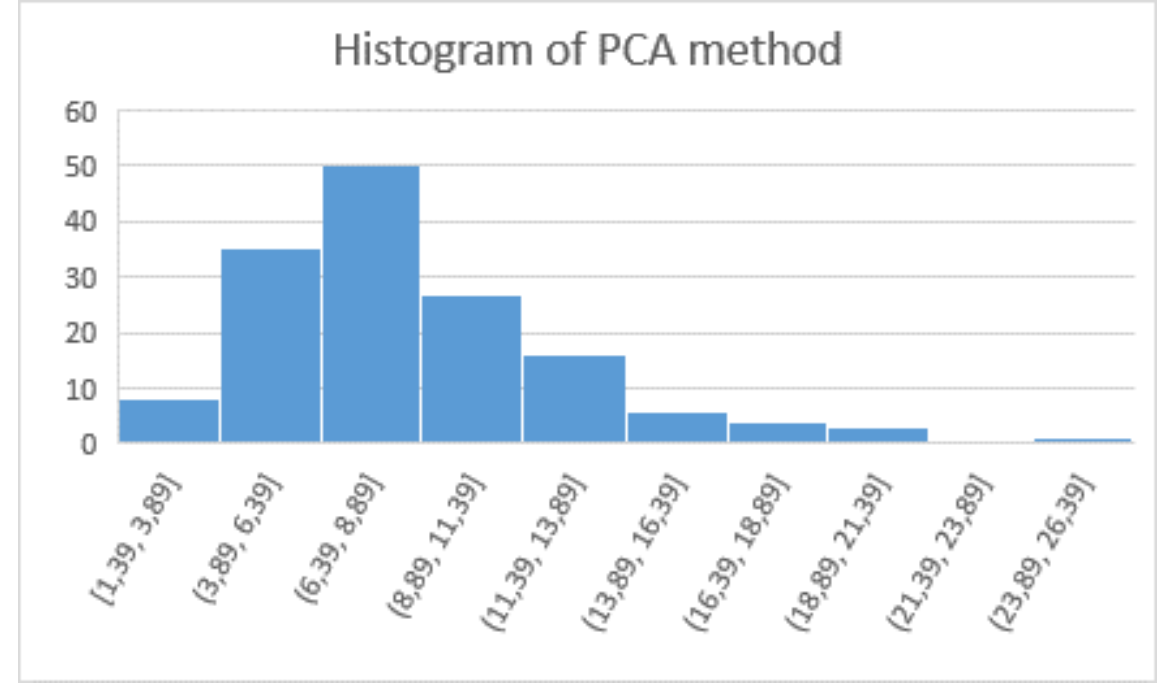

Figure 16. The histogram of PCA method

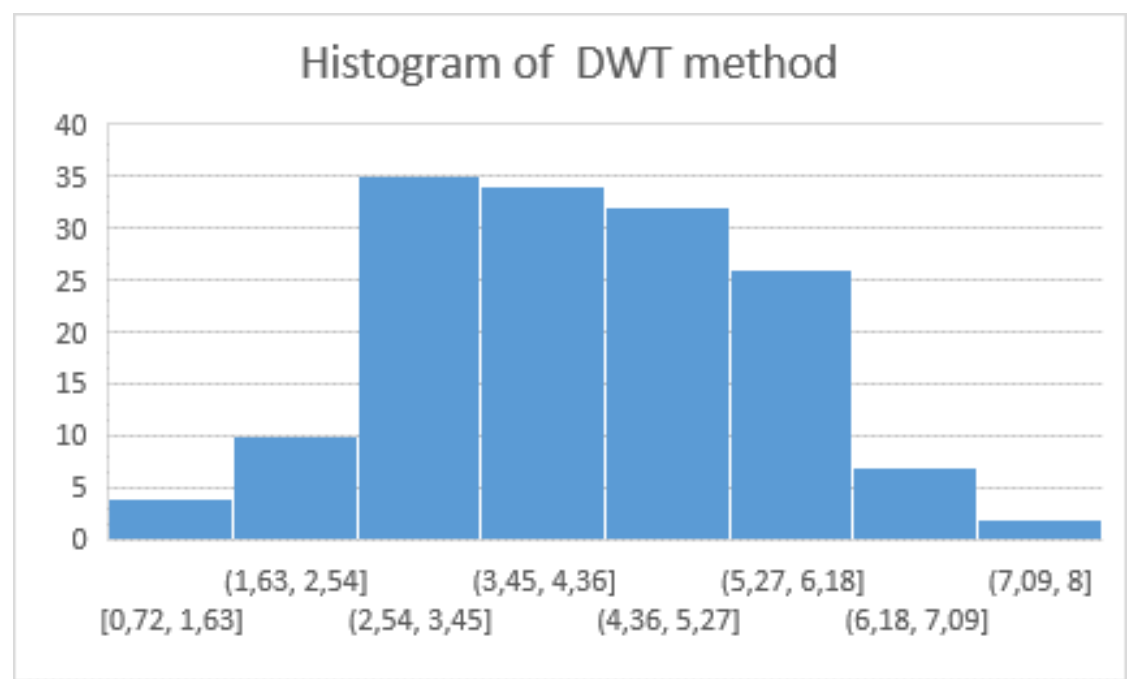

Figure 17. The histogram of DWT method

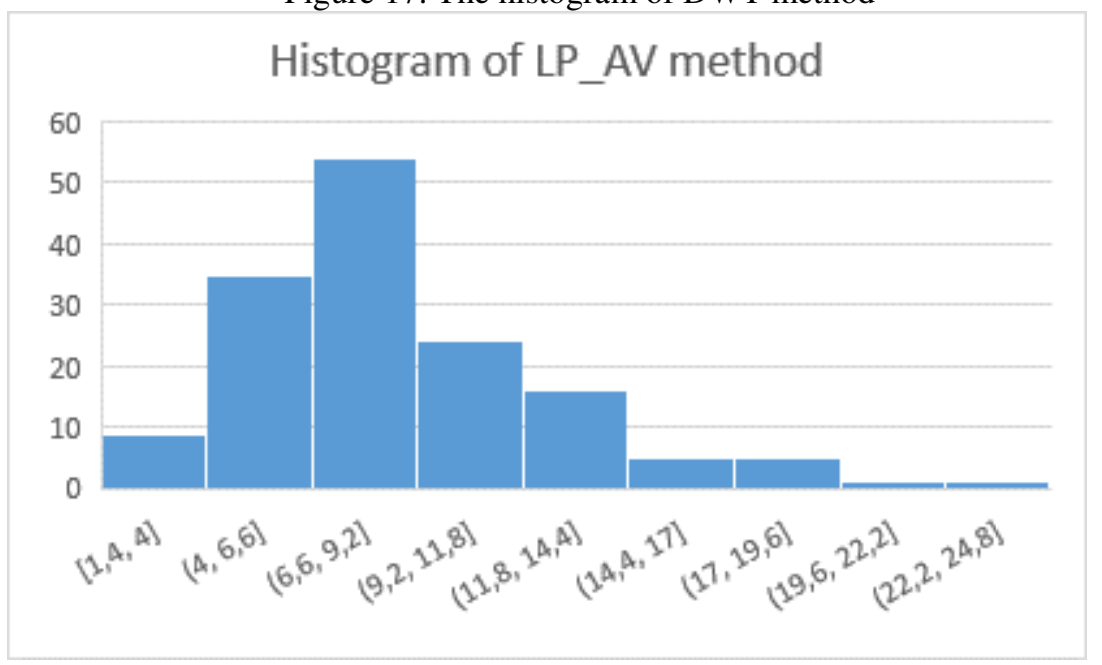

Figure 18. The histogram of LP_AV method 
Signal \& Image Processing: An International Journal (SIPIJ) Vol.11, No.1, February 2020

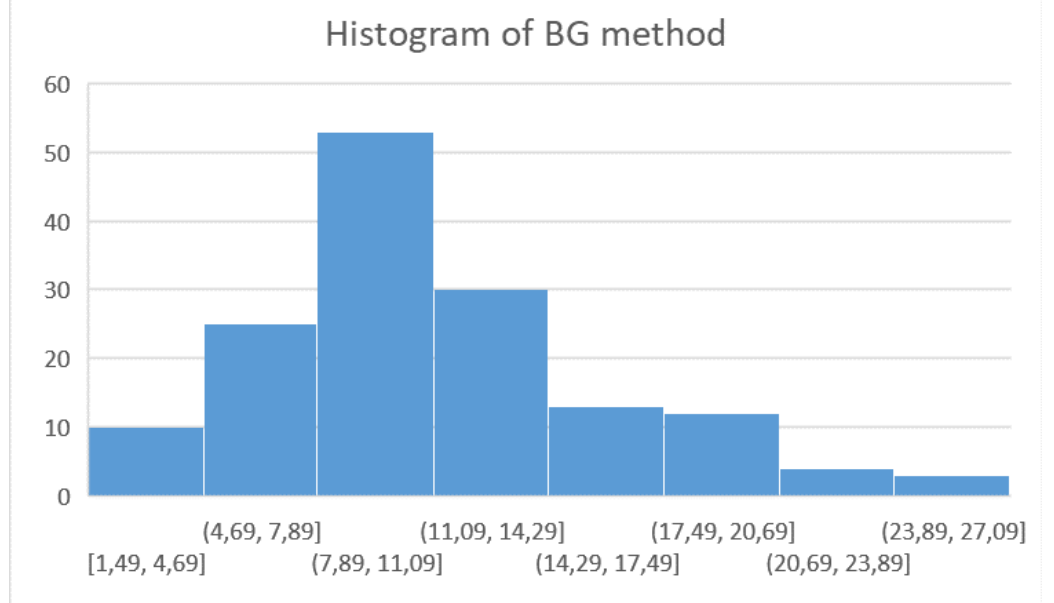

Figure 19. The histogram of LP_PCA method

Now, wecompare analyticallythe proposed method to others methods using the Analysis of variance (ANOVA) with dependent samples (dependence by image). ThesoftwareR gave the following Anova table:

$\begin{array}{lrrrrr} & \text { Df } & \text { Sum Sq } & \text { Mean Sq } & \text { F value } & \text { Pr }(>F) \\ \text { Method } & 5 & 8381 & 1676.2 & 473<2 e-16 \\ \text { Residuals } & 745 & 2640 & 3.5 & & \end{array}$

Since $\operatorname{Pr}(>\mathrm{F})$ wassmaller than $1 \%$, not all methods have significantly identical means. The Newman Keuls test is used to compare the methods in pairs and make groups having significantly the same mean. The software R gave the results below of the test.

$\begin{array}{lrr}\text { \$means } & \text { RMSE } \\ \text { BG } & 11.044867 & 4.859308 \\ \text { DWT } & 4.194067 & 1.381297 \\ \text { LP_AV } & 8.745200 & 3.881025 \\ \text { LP_PCA } & 8.604267 & 3.854875 \\ \text { PCA } & 8.714333 & 3.867235 \\ \text { proposed_M } & 2.158927 & 0.943734 \\ & & \\ \text { \$comparison } & & \\ \text { NULL } & & \\ & & \\ \text { \$groups } & & \\ & & \\ \text { BG } & 11.044867 & \text { groups } \\ \text { LP_AV } & 8.745200 & \mathrm{~b} \\ \text { PCA } & 8.714333 & \mathrm{~b} \\ \text { LP_PCA } & 8.604267 & \mathrm{~b} \\ \text { DWT } & 4.194067 & \mathrm{C} \\ \text { Droposed M } & 2.158927 & \mathrm{~d}\end{array}$

Four different groups: Group "a" contains only method BG having the bigger mean of RMSE (11.045). Group "b" contains 3 methods LP_AV, PCA and LP_PCAthat have significantly the same mean. Group "c" contains only the method DWT which better than group "a" and "b". The 
Signal \& Image Processing: An International Journal (SIPIJ) Vol.11, No.1, February 2020 last group "d" containing the proposed method that the best method because its mean is the smallest by comparing with other means.

\section{Conclusion}

The proposed method of fusion gave better result than other studded methods. The advantage of this method isto use the local fusionwhile taking into account the neighborhood values. This work can be havemany applications, such as:

1. Drone is a new technology in digital imaging, it has opened up unlimited possibilities for enhancing photography. Drone can capture images on the same scene that zooms in on different objects, and at various altitudes. It will produces several images on the same scene but with different objects in-focus.

2. For quality control in food industry, cameras are used to take images. Each camera targets one of several objects to detect an anomaly. The objects are on a treadmill. To have animage containing all the objects in clear we can use the proposed methodwhich would give more details We will use the same technique (alpha stable distance) as a distance to improve Dempster Sheffer method.

\section{REFERENCES}

[1] Jinjiang Li,1,2 Genji Yuan,1,2 and Hui Fan, "Multifocus Image Fusion Using Wavelet-DomainBased Deep CNN", Computational Intelligence and Neuroscience, Vol. 2019, 23 pages, 2019.

[2] K. Xu, Z. Qin, G. Wang, H. Zhang, K. Huang, and S. Ye, "Multi-focus image fusion using fully convolutional two-stream network for visual sensors," KSII Transactions on Internet and Information Systems, Vol. 12, no. 5, pp. 2253-2272, 2018

[3] H. Li, X. He, D. Tao, Y. Tang, and R. Wang, "Joint medical image fusion, denoising and enhancement via discriminative low-rank sparse dictionaries learning," Pattern Recognition, Vol. 79, pp. 130-146, 2018

[4] P. J. Burt and R. J. Kolezynski, "Enhanced image capture through fusion," in Proc. International Conference on Computer Vision,pp. 173-182, 1993.

[5] W. Wang and F. Chang, "A multi-focus image fusion method based on Laplacian pyramid", Journal of Computers [Online].6(12).Available: http://ojs.academypublisher.com/index.php/jcp/article/view/jcp061225592566, Dec. 2011.

[6] P. Zhao, G. Liu, C. Hu, and H. Huang, "Medical image fusion algorithm on the Laplace-PCA," in Proc. Chinese Intelligent Automation Conference, 2013, pp. 787-794, 2013.

[7] R. Sabre, I Wahyuni "Alpha Stable Filter and Distance for Multifocus Image Fusion”, International Journal of Signal Processing Systems, Vol. 7, No. 2, 2019

[8] G. Pajares and J. M. Cruz, "A wavelet-based image fusion tutorial," Pattern Recognition, Vol. 37, pp.1855-1872, 2004.

[9] Q. Guihong, Z. Dali, and Y. Pingfan, "Medical image fusion by wavelet transform modulus maxima". Opt. [Online].9(4).pp.184-190.Available: http://www.opticsinfobase.org/oe/abstract.cfm?uri=oe-9-4-184, 2001.

[10] H. Li, S. Wei, and Y. Chai, "Multifocus image fusion scheme based on feature contrast in the lifting stationary wavelet domain," EURASIP Journal on Advances in Signal Processing, 2012.

[11] Y. Yang, D. Park, S. Huang, and N. Rao, "Medical image fusion via an effective wavelet-based approach," EURASIP Journal an Advances in Signal Processing, 2010.

[12] www.rawsamples.ch. Accessed: 15 November 2017.

[13] T. Wan, N. Canagarajah, and A. Achim, "A Statistical Multiscale Image Segmentation via AlphaStable Modelling," IEEE International Conference on Image Processing, Vol. 4, pp. 357-360, 2017.

[14] Q. Guihong, Z. Dali, and Y. Pingfan, "Medical image fusion by wavelet transform modulus maxima", [Online]. Opt. Express 9(4). pp. 184-190. Available: http://www.opticsinfobase.org/oe/abstract.cfm?uri=oe-9-4-184, 2001. 
Signal \& Image Processing: An International Journal (SIPIJ) Vol.11, No.1, February 2020

[15] F. Maes. "Multimodality image registration by maximization of mutual information", IEEE Transaction Medical Imaging. Vol. 16 Issue 2,1997.

[16] P. J. Burt and E. H. Adelson, "The Laplacian pyramid as a compact image code," IEEE Transactions on Communication, Vol. 31, No. 40, Apr. 1983.

[17] P. J. Burt, "The pyramid as a structure for efficient computation," in Multiresolution Image Processing and Analysis, A. Rosenfeld, Ed., New York: Springer-Verlag, 1984.

[18] I. Wahyuni , "Multi-focus image fusion using local variability", Thèse de doctorat, Université de Bourgogne juin 2017.

[19] S.K. Nayar, "Shape from Focus System,", IEEE Computer Vision and Pattern Recognition, Proceedings CVPR, 1992.

[20] M. Subbarao, "Efficient Depth Recovery through Inverse Optics," Machine Vision for Inspection and Measurement, edited by H. Freeman, Academic Press, 1989.

[21] J. Tian, L. Chen, L. Ma, and W. Yu, "Multi-focus image fusionusing a bilateral gradient-based sharpness criterion,” Optic Communications, Vol. 284, pp. 80-87, 2011.

[22] V.P.S. Naidu, and. J.R. Raol, "Pixel-level Image Fusion using Wavelets and Principal component Analysis”, Defence Science Journal, Vol. 58, No. 3, pp. 338-352. 2008

\section{AUTHORS}

Rachid Sabre received the PhD degree in statistics from the University of Rouen, Rouen, France, in 1993 and Habilitation (HdR) from the University of Burgundy, Dijon, France, in 2003. He joined Agrosup Dijon, Dijon, France, in 1995, where he is an Associate Professor. From 1998 through 2010, he served as a member of Institut de Mathématiques de Bourgogne, France. He was a member of the Scientific Council AgroSup Dijon from 2009 to 2013. In 2012, he has been a member of Laboratoire Electronique, Informatique, et Image (Le2i), France. Since 2019 has been a member of Laboratory Biogeosciences UMR CNRS, University of Burgundy. He is author/co-author of numerous papers in scientific and technical journals and conference proceedings. His research interests lie in areas of statistical process and spectral analysis for signal and image processing.

Ias Sri Wahyuni was born in Jakarta, Indonesia, in 1986. She earned the B.Sc. and M.Sc. degrees in mathematics from the University of Indonesia, Depok, Indonesia, in 2008 and 2011, respectively.In 2009, she joined the Department of Informatic System, Gunadarma University, Depok, Indonesia, as a Lecturer. She is currently a PhD student at University of Burgundy, Dijon, France. Her current research interests include statistics and image processing. 